\begin{tabular}{|c|l|}
\hline Title & Hubble tension in lepton asymmetric cosmology with an extra radiation \\
\hline Author(s) & Osamu, Seto; Yo, Toda \\
\hline Citation & $\begin{array}{l}\text { Physical Review D, 104(6), 063019-1-063019-7 } \\
\text { https://doi.org/10.1103/PhysRevD.104.063019 }\end{array}$ \\
\hline Issue Date & 2021-09-14 \\
\hline Doc URL & http://hdl.handle.net/2115/82983 \\
\hline Rights & @2021 A merican Physical Society \\
\hline Type & article \\
\hline File Information & PhysRevD.104.063019.pdf \\
\hline
\end{tabular}

Instructions for use 


\title{
Hubble tension in lepton asymmetric cosmology with an extra radiation
}

\author{
Osamu Seto ${ }^{1,2, *}$ and Yo Toda ${ }^{2, \dagger}$ \\ ${ }^{1}$ Institute for the Advancement of Higher Education, Hokkaido University, \\ Sapporo 060-0817, Japan \\ ${ }^{2}$ Department of Physics, Hokkaido University, Sapporo 060-0810, Japan
}

(Received 15 April 2021; accepted 15 August 2021; published 14 September 2021)

\begin{abstract}
We study the fit of cosmological models with two additional free parameters $N_{\text {eff }}$ and $\xi_{e}$ in addition to the parameters of $\Lambda \mathrm{CDM}$. We introduce extra radiation components such as hot axions or sterile neutrinos in addition to the energy density of neutrinos with large neutrino degeneracy. Then, a larger $N_{\text {eff }}$ is allowed without spoiling big bang nucleosynthesis (BBN), as positive neutrino degeneracy $\xi_{e}$ could improve BBN fit. By analysing the data from Planck, baryon acoustic oscillation (BAO), BBN and type-Ia supernovae (SNeIa), it can be seen that the Hubble tension can be ameliorated for $\xi_{e} \simeq 0.04$ and $0.3 \lesssim \Delta N_{\text {eff }} \lesssim 0.6$.
\end{abstract}

DOI: 10.1103/PhysRevD.104.063019

\section{INTRODUCTION}

The effective number of relativistic degrees of freedom, $N_{\text {eff }}$, is one of the simplest extension of the standard cosmology and effectively constrains an extension of the standard model (SM) of particle physics. Since the standard model of cosmology, the $\Lambda \mathrm{CDM}$ model with the cosmological constant $\Lambda$ as the dark energy and cold dark matter (CDM), has been successful, $N_{\text {eff }}$ has been well constrained by many cosmological observations. Planck (2018) [1] has reported the constraint as $N_{\text {eff }}=2.99 \pm 0.17(68 \%)$ based on the combined data of cosmic microwave background (CMB) and baryon acoustic oscillation (BAO). The combined data on abundance of light elements synthesized at the big bang nucleosynthesis (BBN) and CMB also provide $N_{\text {eff }}=2.88 \pm 0.16(1 \sigma)$ [2]. Those are consistent with the prediction of the SM of particle physics, $N_{\mathrm{eff}}^{\mathrm{SM}} \simeq 3.046$ [3]. For some pioneering works and recent progress of this calculation, see, e.g., Refs. [4-7] and Refs. [8-12], respectively.

Interestingly, a somewhat larger $N_{\text {eff }}$ is also indicated by the discrepancy between the measured values of the Hubble parameter by local (low red shift) measurements and that by distant (high red shift) measurements. Assuming $\Lambda \mathrm{CDM}$ model, Planck measurements of CMB anisotropy infers $H_{0}=67.4 \pm 0.5 \mathrm{~km} / \mathrm{s} / \mathrm{Mpc}[1]$. Other distant observations such as the Atacama Cosmology Telescope [13], BAO [14], and the combination of $\mathrm{BAO}+\mathrm{BBN}$ analysis (independent of CMB) [15] all infer $H_{0} \sim 67 \mathrm{~km} / \mathrm{s} / \mathrm{Mpc}$. On the other hand, local measurements of $H_{0}$ by the SHOES collaboration with Cepheids and type Ia supernovae (SNe Ia) in Ref. [16]

*seto@particle.sci.hokudai.ac.jp

y-toda@particle.sci.hokudai.ac.jp (hereafter, R18) and Ref. [17] (hereafter R19) and by the HOLiCOW collaboration with lensed quasars [18] have reported as $H_{0} \sim 73 \mathrm{~km} / \mathrm{s} / \mathrm{Mpc}$. Another local measurement using the Tip of the Red Giant Branch (TRGB) as distance ladders has obtained a value between Planck and the SHOES, $H_{0} \sim 70 \mathrm{~km} / \mathrm{s} / \mathrm{Mpc}$ [19].

Until now, numerous attempts have been made to this problem. See, e.g., Ref. [20] for a recent review. One of the simplest approaches is to introduce additional relativistic degrees of freedom $\Delta N_{\text {eff }}=N_{\text {eff }}-N_{\text {eff }}^{\mathrm{SM}}$. This is because that the shorten sound horizon $r_{s *}$ at the recombination epoch by the extra energy density with a larger $N_{\text {eff }}$ and the measured angular size $\theta_{*} \equiv r_{*} / D_{M *}$ of the acoustic scale infers a shorter angular diameter distance $D_{M *} \propto 1 / H_{0}$. The preferred value has been suggested as $0.2 \lesssim \Delta N_{\text {eff }} \lesssim$ $0.5(\mathrm{CMB}+\mathrm{BAO}+\mathrm{R} 18)[1]$ and

$$
0.2 \lesssim \Delta N_{\text {eff }} \lesssim 0.4
$$

$(\mathrm{CMB}+\mathrm{BAO}+$ Panthenon [21] + R19+ BBN $)$ [22], depending upon datasets. Such an extra $\Delta N_{\text {eff }}$ can be easily realized in various particle physics beyond the SM such as hot axion [23] or the $L_{\mu}-L_{\tau}$ gauge interaction [24]. However, as mentioned above, the magnitude of $N_{\text {eff }}$ is constrained by various reasons, principally, $\mathrm{CMB}, \mathrm{BBN}$ and large scale structure of the Universe. This BBN limit primarily comes from the Helium mass fraction $Y_{P}$ that constrains the extra energy density not to speed up the cosmic expansion too much. Actually, there is a possibility to relax the constraint by $Y_{P}$ significantly. If our Universe has a large electron-type lepton asymmetry $\xi_{e}$, which could suppress the conversion of proton to neutron, the resultant $Y_{P}$ can be consistent with the observation for a somewhat large $N_{\text {eff }}$ [25-33]. Various mechanisms for generating a large lepton asymmetry have been proposed in literature 
[34-41]. In those scenarios, for example, a large lepton asymmetry has been generated after the electroweak symmetry breaking or in thermal history with no electroweak symmetry restoration after inflation, with generating the right magnitude of the baryon asymmetry.

In this paper, we investigate the fit of cosmological models with additional two free parameters $N_{\text {eff }}$ and $\xi_{e}$ besides $\Lambda \mathrm{CDM}$ parameters. We consider that lepton asymmetry as well as extra radiation components in addition to the contribution from neutrino degeneracy given by the following Eq. (6) are independent free parameters. This is the essential ingredient in this work. In fact, previously, it has been pointed out that the $\Delta N_{\text {eff }}$ from lepton asymmetry is not effective to alleviate the Hubble tension in Ref. [32], which is quoted at Sec. 7.2 in Ref. [20] as well. In the paper [32], it has been assumed that all $\Delta N_{\text {eff }}$ are originated from lepton asymmetry and the electron-type lepton asymmetry is negligibly tiny to keep BBN intact. In contrast to those previous works $[32,42]$, by taking account of the effect by the electron-type lepton asymmetry to the Helium abundance, we evaluate the fitting to various data, principally including $\mathrm{CMB}, \mathrm{BAO}$ and $\mathrm{BBN}$ and show that some amount of electron-type lepton asymmetry of the order of $10^{-2}$ with an extra radiation is promising.

\section{COSMOLOGICAL EFFECTS OF $N_{\text {eff }}$ AND $\xi_{e}$}

The extra radiation affects not only $\mathrm{CMB}$ but also $\mathrm{BBN}$, because the additional component increases the expansion rate of the Universe, the decoupling temperature of the weak interaction, and the neutron-to-proton ratio. The increased neutron-to-proton ratio results in the larger helium mass fraction. The measured Helium mass fraction $Y_{P}=0.2449 \pm 0.0040$ [43] does not appear to be compatible with a large extra $N_{\text {eff }}$.

To let a larger $N_{\text {eff }}$ available, we introduce non-negli gible lepton asymmetry. We use the degeneracy parameter

$$
\xi_{i}=\frac{\mu_{\nu_{i}}}{T_{\nu}},
$$

to parametrize the lepton asymmetry, where $\mu_{\nu_{i}}$ is the chemical potential for $i(=e, \mu, \tau)$ th flavor neutrino and $T_{\nu}$ is the temperature of neutrinos. The Fermi-Dirac distribution functions for $i$ th flavor (anti)neutrinos with nonvanishing neutrino degeneracy is expressed as

$$
\begin{aligned}
f_{\nu_{i}}\left(p, \xi_{i}\right) & =\frac{1}{\exp \left(\frac{p}{T_{\nu}}-\xi_{i}\right)+1}, \\
f_{\bar{\nu}_{i}}\left(p, \xi_{i}\right) & =\frac{1}{\exp \left(\frac{p}{T_{\nu}}+\xi_{i}\right)+1},
\end{aligned}
$$

with $p$ being the proper momentum. The lepton asymmetry $\xi_{i}$ affects both BBN and CMB as (principally) following three ways $[25,26,28]$.
First, the lepton asymmetry is the difference between the number of particles and antiparticles. From our definition of the lepton asymmetry, there are more neutrinos than antineutrinos in the positive asymmetric Universe. In particular, the electron-type lepton asymmetry

$$
L_{e} \equiv \frac{n_{\nu_{e}}-n_{\bar{\nu}_{e}}}{n_{\gamma}}=\frac{1}{36 \zeta(3)}\left(\frac{T_{\nu_{e}}}{T_{\gamma}}\right)^{3}\left(\pi^{2} \xi_{\nu_{e}}+\xi_{\nu_{e}}^{3}\right),
$$

is interesting. At the beginning of the $\mathrm{BBN}$, neutrons and protons are in equilibrium until the decoupling of the weak interaction. If there is sizable positive $\xi_{e}$, the process $p+\bar{\nu}_{e} \rightarrow n+e^{+}$is suppressed compared with the process $n+\nu_{e} \rightarrow p+e^{-}$because of less antielectron neutrinos. With such a suppressed neutron-to-proton ratio, the resulting Helium mass fraction $Y_{P}$ is decreased. This may compensate for the $Y_{P}$ increase caused by a larger $N_{\text {eff }}$. Although we do not deal degeneracy parameters of other flavors $\xi_{\mu}$ and $\xi_{\tau}$ as explicit input parameters, it is expected that all of those are of the same order of magnitudes as $\xi_{\mu} \sim \xi_{\tau} \sim \xi_{e}$ because of neutrino oscillation [44-48].

Second, the energy density of neutrinos is increased by the neutrino degeneracy as

$$
\begin{gathered}
\rho_{\nu+\bar{\nu}}=\sum_{i} T_{\nu}^{4} \int \frac{d^{3} p}{(2 \pi)^{3}} p\left[f_{\nu_{i}}\left(p, \xi_{i}\right)+f_{\bar{\nu}_{i}}\left(p, \xi_{i}\right)\right] \\
=\left.\rho_{\nu+\bar{\nu}}\right|_{\xi=0}+\Delta \rho_{\nu+\bar{\nu}}(\xi), \\
\Delta \rho_{\nu+\bar{\nu}} \propto T_{\nu}^{4}\left(\sum_{\xi_{i}} \xi_{i}^{2}+\mathcal{O}\left(\xi_{i}^{4}\right)\right),
\end{gathered}
$$

with $\left.\rho_{\nu+\bar{\nu}}\right|_{\xi=0}$ being the energy density with vanishing $\xi_{i}$. However, for small degeneracy as $\xi<0.1$ as we will consider, the extra contribution to the energy density $\Delta \rho_{\nu+\bar{\nu}}(\xi)$ from the neutrino degeneracy $\xi$ is very small for both BBN and CMB. The $\xi$ dependent part of $\Delta N_{\text {eff }}$, $\Delta N_{\text {eff }}(\xi)$, is $\mathcal{O}\left(10^{-2}\right)$. In any case, given that we are introducing an extra radiation (ER) component, one may regard our $N_{\text {eff }}$ as $\Delta N_{\text {eff }}=\Delta N_{\text {eff }}^{\mathrm{ER}}+\Delta N_{\text {eff }}(\xi)$.

Third, in perturbation level, the asymmetry factor comes into the source term of the Boltzmann equation. This is given in the synchronous gauge by [49],

$$
\frac{\partial \Psi}{\partial \tau}+i(\boldsymbol{k} \cdot \hat{n}) \Psi+\frac{d \ln \left(f_{\nu}+f_{\bar{\nu}}\right)}{d \ln q}\left[\dot{\eta}-\frac{\dot{h}+6 \dot{\eta}}{2}(\hat{k} \cdot \hat{n})^{2}\right]=0,
$$

with $q=a p$. Here, $\Psi$ is the perturbation to the distribution function, $\hat{n}$ is the direction of the momentum, $\tau$ is the conformal time, $\boldsymbol{k}$ is the wave number of the Fourier mode, and $h$ and $\eta$ are the synchronous metric perturbations. In the computation of the power spectrum $C_{l}$, when we integrate Eq. (7) with $\left(f_{\nu}+f_{\bar{\nu}}\right) q^{3} d q$, this $\xi$ dependence in 


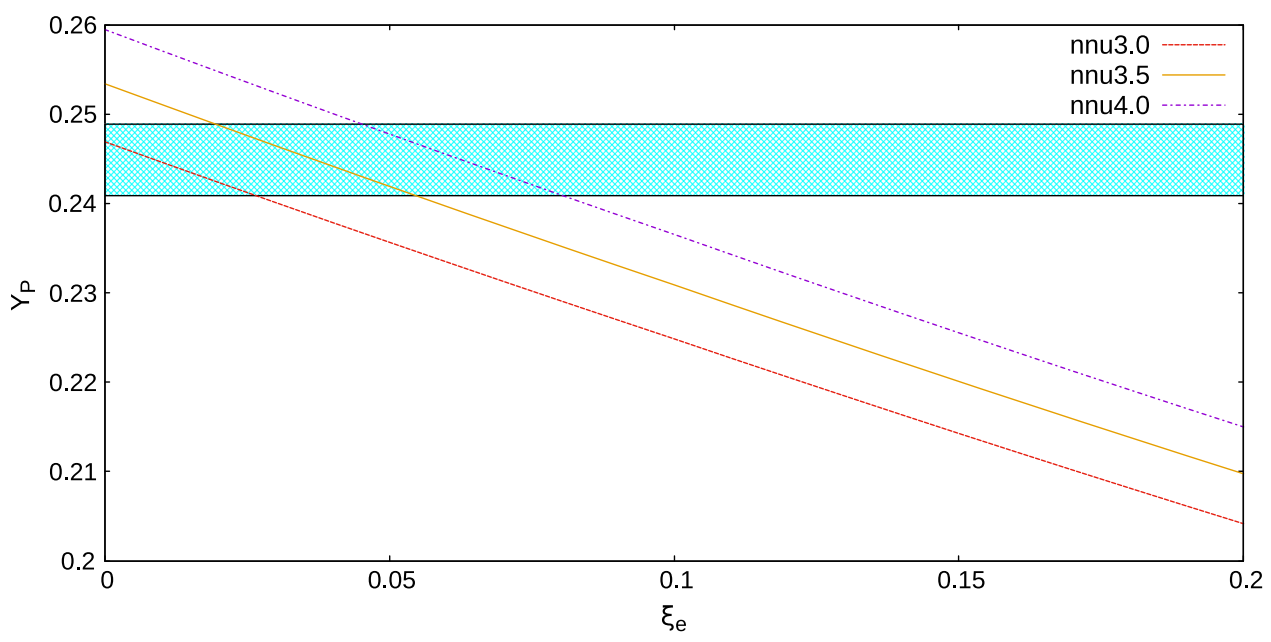

FIG. 1. Effects of electron-type lepton asymmetry $\xi_{e}$ and the relativistic degrees of freedom $N_{\text {eff }}$ on the helium abundance $Y_{P}$. A larger $N_{\text {eff }}$ leads to a larger $Y_{P}$, while a larger $\xi_{e}$ suppresses the resulting $Y_{P}$. The shading rectangle illustrates the value of the $Y_{P}$ measurement: $Y_{P}=0.2449 \pm 0.0040[43]$.

perturbation disappears [26]. Accordingly, Eq. (7) applies in nonvanishing $\xi$ cases without any change.

\section{DATA AND ANALYSIS}

We perform a Markov-Chain Monte Carlo (MCMC) analysis on a $N_{\text {eff }}$ model with a large lepton asymmetry. We use the public MCMC code CosmoMCplanck2 018 [50]. For estimation of light elements with nonvanishing lepton degeneracy $\xi_{e}$, we have also used PArthENOPE2.0-Standard [51]. Since a small Hubble parameter is indicated if we allow larger neutrino masses [1], in order to minimize this effect, we assume the smallest neutrino mass with the spectrum of normal hierarchy, $m_{\nu}=(0,0,0.06) \mathrm{eV}$, in this paper.

\section{A. Datasets}

We analyze the models by referring to the following cosmological observation data sets. We include both temperature and polarization likelihoods for high $l \mathrm{plik}$ ( $l=30$ to 2508 in TT and $l=30$ to 1997 in $\mathrm{EE}$ and TE) and low $l$ Commander and lowE SimAll ( $l=2$ to 29$)$ of Planck (2018) measurement of the CMB temperature anisotropy [1]. We also include Planck lensing [52]. For constraints on low red shift cosmology, we include data of BAO from 6dF [53], DR7 [54], and DR12 [55]. We also include Pantheon [21] of the local measurement of light curves and luminosity distance of supernovae as well as SH0ES (R19) [17] of the local measurement of the Hubble constant from the Hubble Space Telescope observation of Supernovae and Cepheid variables. Finally, we include the data sets of helium mass fraction $Y_{P}$ measurement [43] and deuterium abundance $D / H$ measurement [56] to impose BBN constraints.

\section{RESULT AND DISCUSSION}

We show, in Fig. 1, a calculated $Y_{P}$ for various $N_{\text {eff }}, \xi_{e}$ and a fixed baryon asymmetry $\Omega_{b} h^{2}=0.223$. A larger $N_{\text {eff }}$ leads to larger $Y_{P}$, while a larger $\xi_{e}$ leads to smaller $Y_{P}$ $[27,28]$. The asymmetry $\xi_{e} \simeq 0.03$ is capable of canceling $\Delta N_{\text {eff }} \simeq 0.5$ contribution to $Y_{P}$. We find that $\xi_{e} \lesssim 0.03$ for $N_{\text {eff }}=3.0,0.02 \lesssim \xi_{e} \lesssim 0.06$ for $N_{\text {eff }}=3.5$, and $0.05 \lesssim$ $\xi_{e} \lesssim 0.08$ for $N_{\text {eff }}=4.0$ are consistent with the $Y_{P}$ measurement.

Electron lepton asymmetry $\xi_{e}$ decreases slightly $D / H$. In the fit to the CMB data, there is some correlation or parameter degeneracy between $\Omega_{b} h^{2}$ and $\Delta N_{\text {eff }}$. The simultaneous increase of $\Delta N_{\text {eff }}$ and $\Omega_{b} h^{2}$ does not alter the resultant $D / H$ abundance $[22,51,57]$.

The Monte Carlo analysis has been carried out using datasets $(\mathrm{CMB}+\mathrm{BAO}+\mathrm{BBN}+\mathrm{JLA}+\mathrm{R} 19)$. The posteriors of parameters are summarized in Fig. 2. We show the results of four different neutrino degeneracy parameters $\xi_{e}=0,0.02,0.04$, and 0.06 . As $\xi_{e}$ increases, a larger $N_{\text {eff }}$ and, as the results, $H_{0}$ are allowed and the predicted $Y_{P}$ decreases. With the change in $Y_{P}, \chi^{2}$ for the $Y_{P}$ measurement is minimized for $\xi_{e} \sim 0.04$.

The best-fit values of some quantities and the values of $\chi^{2}$ for the best-fit points of $N_{\text {eff }}$ models with several values of electron-type lepton degeneracy $\xi_{e}$ are summarized in Table I. The total $\chi^{2}$ is also minimized at $\xi_{e} \simeq 0.04$ for two reasons. One is that, just mentioned above, light elements observations prefer $\xi_{e} \sim 0.04\left(\chi_{\text {Abund }}^{2}=\chi_{\text {Cooke17 }}^{2}+\chi_{\text {Aver15 }}^{2}\right.$ at $\xi_{e}=0.04$ is about one-ninth of at $\xi_{e}=0.00$ ). The other reason is that the above datasets include R19, which indicates a larger $H_{0}$. When we introduce large asymmetry (e.g., $\xi_{e}=0.06$ ), a larger $N_{\text {eff }}$ is required, then the Hubble tension could be relaxed greatly. However, such a too large $N_{\text {eff }}$ is hardly compatible with both CMB and BBN. 


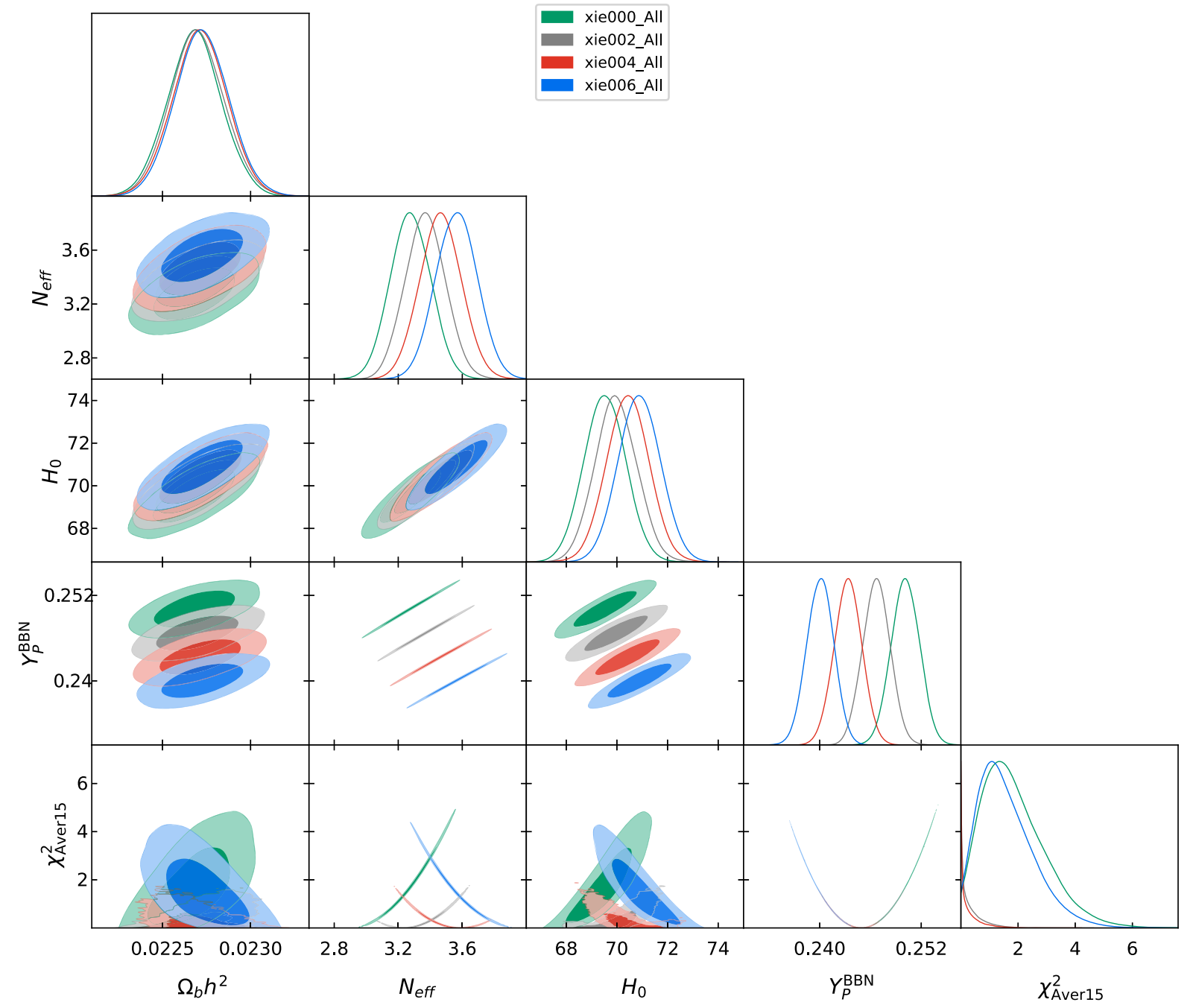

FIG. 2. Posterior distributions of $\Omega_{b} h^{2}, N_{\text {eff }}, H_{0}, Y_{P}$, and $\chi_{\text {Aver }}^{2}$ on a $N_{\text {eff }}$ model for several cases with different amount of lepton asymmetry. This posterior have been derived for all datasets (CMB + BAO + JLA + BBN + R19).

TABLE I. The best-fit $\chi^{2}$ for a $N_{\text {eff }}$ model with and without a lepton asymmetry. Here, for reference, we note that the value of $\chi_{\text {total }}^{2}=3841.52$ for $\Lambda$ CDM under the same dataset has been derived [22].

\begin{tabular}{lcccc}
\hline \hline Parameter & $\xi_{e}=0.00$ & $\xi_{e}=0.02$ & $\xi_{e}=0.04$ & $\xi_{e}=0.06$ \\
\hline$N_{\text {eff }}$ & 3.243 & 3.313 & 3.455 & 3.634 \\
$H_{0}[\mathrm{~km} / \mathrm{s} / \mathrm{Mpc}]$ & 69.632 & 69.716 & 70.258 & 71.701 \\
$Y_{P}$ & 0.250 & 0.246 & 0.243 & 0.241 \\
$\chi_{\text {Cooke17 }}^{2}$ & 0.10 & 0.06 & $1.5 \times 10^{-3}$ & $6.6 \times 10^{-6}$ \\
$\chi_{\text {Aver15 }}^{2}$ & 1.45 & 0.08 & 0.17 & 0.97 \\
$\chi_{\text {H074p03 }}^{2}$ & 9.59 & 9.23 & 7.06 & 2.69 \\
$\chi_{\text {JLA }}^{2}$ & 1034.74 & 1034.74 & 1034.75 & 1034.81 \\
$\chi_{\text {prior }}^{2}$ & 4.31 & 2.31 & 3.20 & 7.23 \\
$\chi_{\text {CMB }}^{2}$ & 2781.60 & 2783.90 & 2782.84 & 2783.71 \\
$\chi_{\text {BAO }}^{2}$ & 5.80 & 5.41 & 5.38 & 6.57 \\
$\chi_{\text {total }}^{2}$ & 3837.61 & 3835.72 & 3833.39 & 3836.22 \\
\hline \hline
\end{tabular}

Therefore, we conclude that the most preferred electron lepton asymmetry is $\xi_{e}=0.04$. This is consistent with the previous works: $\xi=-0.002_{-0.111}^{+0.114}(95 \%)$ in Ref. [58]. ${ }^{1} \mathrm{We}$ obtain

$$
\begin{aligned}
N_{\text {eff }}= & 3.46 \pm 0.13 \\
H_{0}= & 70.43 \pm 0.84 \mathrm{~km} / \mathrm{s} / \mathrm{Mpc}, \\
\text { for } \xi_{e}= & 0.04(68 \%, \text { Planck }+\mathrm{BAO}+\text { Panthenon } \\
& +\mathrm{R} 19+\mathrm{BBN}) .
\end{aligned}
$$

The values (8) show that the mean central value exceeds $H_{0}=70.0 \mathrm{~km} / \mathrm{s} / \mathrm{Mpc}$ in $\xi_{e}=0.04$ cosmology. The best-

\footnotetext{
${ }^{1}$ Prospects in the future measument CMB-S4 have been studied in Ref. [59].
} 


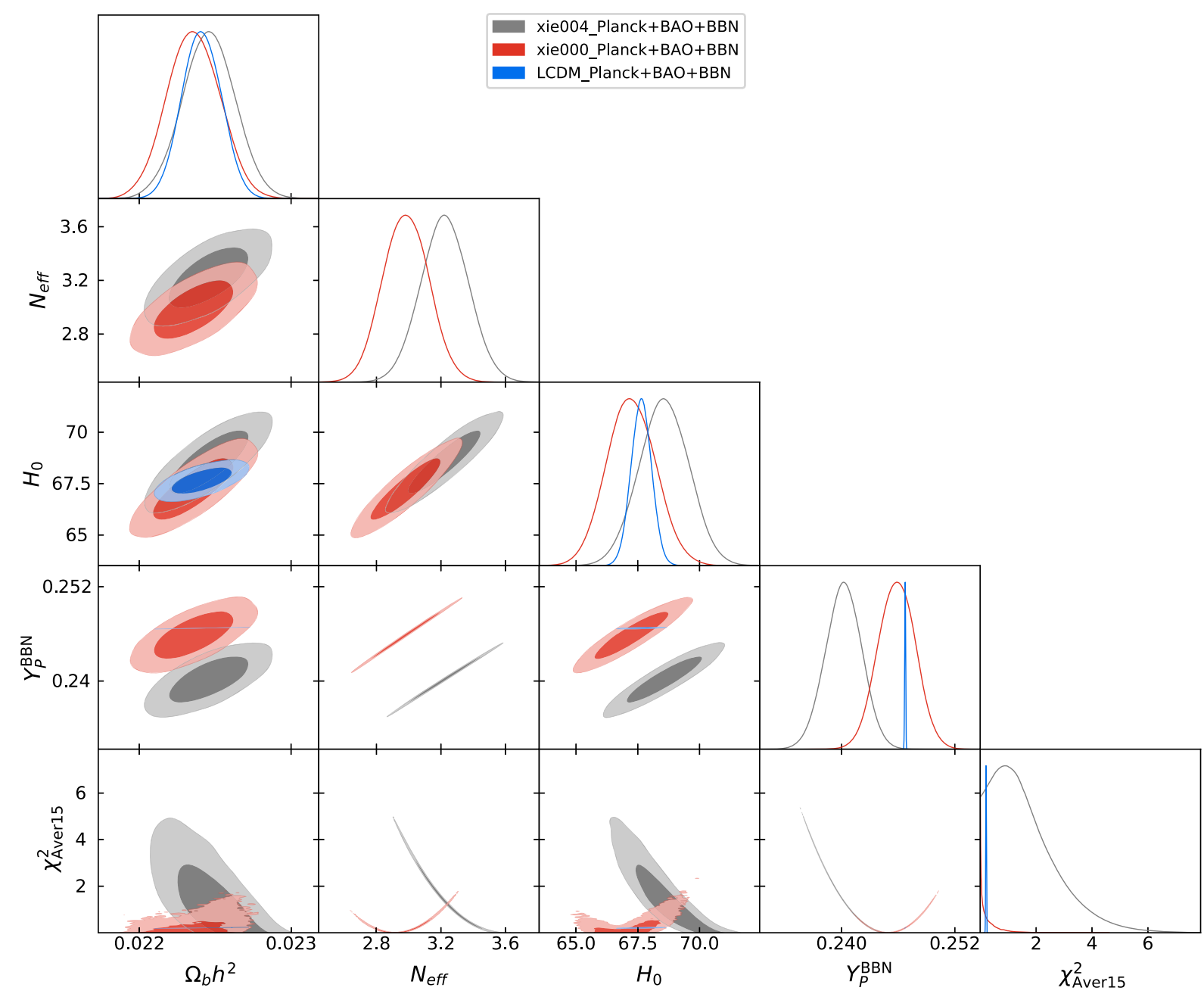

FIG. 3. Same as Fig. 2 for distant datasets $(\mathrm{CMB}+\mathrm{BAO}+\mathrm{BBN})$ only.

fit value is displayed in Table I. By comparing the values (8) with the previous ones (1) for vanishing $\xi$ based on the same dataset, we find favored values of $\Delta N_{\text {eff }}$ is significantly shifted as $0.3 \lesssim \Delta N_{\text {eff }} \lesssim 0.6$ due to the lepton asymmetry.

We have also performed the analysis with the only distant data sets: Planck $+\mathrm{BAO}+\mathrm{BBN}$ at $\xi_{e}=0.04$, which is the best-fit point for the combination of local and distant observations. The result is shown in Fig. 3 with $\Lambda \mathrm{CDM}$ and $N_{\text {eff }}$ without any neutrino degeneracy for comparison. We obtain

$$
\begin{aligned}
N_{\text {eff }}= & 3.22 \pm 0.29, \\
H_{0}= & 68.6 \pm 2.0 \mathrm{~km} / \mathrm{s} / \mathrm{Mpc}, \quad \text { for } \xi_{e}=0.04 \\
& (95 \%, \text { Planck }+\mathrm{BAO}+\mathrm{BBN}) .
\end{aligned}
$$

For comparison, we note

$$
\begin{aligned}
N_{\text {eff }}= & 2.98_{-0.27}^{+0.28}, \\
H_{0}= & 67.2_{-1.9}^{+2.0} \mathrm{~km} / \mathrm{s} / \mathrm{Mpc}, \quad \text { for } \xi_{e}=0.00 \\
& (95 \%, \text { Planck }+\mathrm{BAO}+\mathrm{BBN}),
\end{aligned}
$$

and quote $N_{\text {eff }}=2.89 \pm 0.29(95 \%$, Planck + BBN $)$ from the Planck paper [1]. As shown in Fig. 3, the value of $H_{0}=$ $70.0 \mathrm{~km} / \mathrm{s} / \mathrm{Mpc}$ lies in the uncertainty for the best-fit asymmetry $\xi_{e}=0.04$, even if we refer only distant observations.

\section{SUMMARY}

We have studied cosmology with both the extra radiation parametrized by $N_{\text {eff }}$ and a large lepton asymmetry, and examined the fit to various cosmological data. In contrast to Ref. [32], we consider the cases that an additional contribution, to $N_{\text {eff }}$ beside that comes from the neutrino degeneracy $\xi$, such as hot axions and right-handed neutrinos although we do not need to specify species. 
We have shown that a larger $N_{\text {eff }}$ and $H_{0}$ are indicated for a larger neutrino degeneracy $\xi_{e}$. At the best fit point with $\xi_{e}=0.04$ by taking all $\mathrm{BBN}, \mathrm{CMB}, \mathrm{BAO}$ and local measurements into account, the total $\chi^{2}$ of lepton asymmetric cosmology can be reduced by about 4 from that of $N_{\text {eff }}$ and by about 8 from that of $\Lambda$ CDM model. We conclude that $\xi_{e} \simeq 0.04$ and $0.3 \lesssim \Delta N_{\text {eff }} \lesssim 0.6$ are favored to ameliorate the Hubble tension. The construction of such a model of particle physics able to reproduce these values would be worth examining.

Even if we analyze only the distant cosmological data of Planck, BAO, and BBN, we can find a clear difference between the $N_{\text {eff }}+\xi_{e}$ models and the $\Lambda$ CDM. For $\xi_{e}=0.04$, the data from Planck, BAO, and BBN also infer significantly larger $H_{0}$ and $N_{\text {eff }}$. Then, $H_{0}=$ $70.0 \mathrm{~km} / \mathrm{s} / \mathrm{Mpc}$ is within the uncertainty, hence we might claim that, at least, the tension between the Planck measurement and local $H_{0}$ measurements with TRGB calibration could be resolved by a large cosmological lepton asymmetry.

\section{ACKNOWLEDGMENTS}

This work is supported in part by the Japan Society for the Promotion of Science (JSPS) KAKENHI Grants No. 19K03860, No. 19K03865 and No. 21H00060 (O. S.).
[1] N. Aghanim et al. (Planck Collaboration), Astron. Astrophys. 641, A6 (2020).

[2] R. H. Cyburt, B. D. Fields, K. A. Olive, and T. H. Yeh, Rev. Mod. Phys. 88, 015004 (2016).

[3] G. Mangano, G. Miele, S. Pastor, T. Pinto, O. Pisanti, and P. D. Serpico, Nucl. Phys. B729, 221 (2005).

[4] S. Dodelson and M. S. Turner, Phys. Rev. D 46, 3372 (1992).

[5] A. D. Dolgov and M. Fukugita, Phys. Rev. D 46, 5378 (1992).

[6] R. E. Lopez, S. Dodelson, A. Heckler, and M. S. Turner, Phys. Rev. Lett. 82, 3952 (1999).

[7] A. D. Dolgov, S. H. Hansen, and D. V. Semikoz, Nucl. Phys. B543, 269 (1999).

[8] M. Escudero, J. Cosmol. Astropart. Phys. 02 (2019) 007.

[9] J. J. Bennett, G. Buldgen, M. Drewes, and Y. Y. Y. Wong, J. Cosmol. Astropart. Phys. 03 (2020) 003.

[10] M. E. Abenza, J. Cosmol. Astropart. Phys. 05 (2020) 048.

[11] K. Akita and M. Yamaguchi, J. Cosmol. Astropart. Phys. 08 (2020) 012.

[12] J. J. Bennett, G. Buldgen, P. F. De Salas, M. Drewes, S. Gariazzo, S. Pastor, and Y. Y. Y. Wong, J. Cosmol. Astropart. Phys. 04 (2021) 073.

[13] S. Aiola et al. (ACT Collaboration), J. Cosmol. Astropart. Phys. 12 (2020) 047.

[14] G. E. Addison, D. J. Watts, C. L. Bennett, M. Halpern, G. Hinshaw, and J. L. Weiland, Astrophys. J. 853, 119 (2018).

[15] N. Schöneberg, J. Lesgourgues, and D. C. Hooper, J. Cosmol. Astropart. Phys. 10 (2019) 029.

[16] A. G. Riess et al., Astrophys. J. 855, 136 (2018).

[17] A. G. Riess, S. Casertano, W. Yuan, L. M. Macri, and D. Scolnic, Astrophys. J. 876, 85 (2019).

[18] K. C. Wong, S. H. Suyu, G. C. F. Chen, C. E. Rusu, M. Millon, D. Sluse, V. Bonvin, C. D. Fassnacht, S. Taubenberger, M. W. Auger et al., Mon. Not. R. Astron. Soc. 498, 1420 (2020).

[19] W. L. Freedman, B. F. Madore, D. Hatt, T. J. Hoyt, I. S. Jang, R. L. Beaton, C. R. Burns, M. G. Lee, A. J. Monson, J. R. Neeley et al., Astrophys. J. 882, 34 (2019).
[20] E. Di Valentino, O. Mena, S. Pan, L. Visinelli, W. Yang, A. Melchiorri, D. F. Mota, A. G. Riess, and J. Silk, Classical Quantum Gravity 38, 153001 (2021).

[21] D. M. Scolnic, D. O. Jones, A. Rest, Y. C. Pan, R. Chornock, R. J. Foley, M. E. Huber, R. Kessler, G. Narayan, A. G. Riess et al., Astrophys. J. 859, 101 (2018).

[22] O. Seto and Y. Toda, Phys. Rev. D 103, 123501 (2021).

[23] F. D’Eramo, R. Z. Ferreira, A. Notari, and J. L. Bernal, J. Cosmol. Astropart. Phys. 11 (2018) 014.

[24] M. Escudero, D. Hooper, G. Krnjaic, and M. Pierre, J. High Energy Phys. 03 (2019) 071.

[25] G. Beaudet and P. Goret, Astron. Astrophys. 49, 415 (1976), http://articles.adsabs.harvard.edu/pdf/1976A\%26A. ...49..415B.

[26] J. Lesgourgues and S. Pastor, Phys. Rev. D 60, 103521 (1999).

[27] P. D. Serpico and G. G. Raffelt, Phys. Rev. D 71, 127301 (2005).

[28] M. Shiraishi, K. Ichikawa, K. Ichiki, N. Sugiyama, and M. Yamaguchi, J. Cosmol. Astropart. Phys. 07 (2009) 005.

[29] D. Kirilova, Hyperfine Interact. 215, 111 (2013).

[30] A. Caramete and L. A. Popa, J. Cosmol. Astropart. Phys. 02 (2014) 012.

[31] C. T. Yang, J. Birrell, and J. Rafelski, arXiv:1812.05157.

[32] G. Barenboim, W. H. Kinney, and W. I. Park, Eur. Phys. J. C 77, 590 (2017).

[33] G. B. Gelmini, M. Kawasaki, A. Kusenko, K. Murai, and V. Takhistov, J. Cosmol. Astropart. Phys. 09 (2020) 051.

[34] R. Foot, M. J. Thomson, and R. R. Volkas, Phys. Rev. D 53, R5349 (1996).

[35] X. D. Shi, Phys. Rev. D 54, 2753 (1996).

[36] A. Casas, W. Y. Cheng, and G. Gelmini, Nucl. Phys. B538, 297 (1999).

[37] J. McDonald, Phys. Rev. Lett. 84, 4798 (2000).

[38] M. Kawasaki, F. Takahashi, and M. Yamaguchi, Phys. Rev. D 66, 043516 (2002).

[39] M. Yamaguchi, Phys. Rev. D 68, 063507 (2003). 
[40] F. Takahashi and M. Yamaguchi, Phys. Rev. D 69, 083506 (2004).

[41] M. Shaposhnikov, J. High Energy Phys. 08 (2008) 008.

[42] R. C. Nunes and A. Bonilla, Mon. Not. R. Astron. Soc. 473, 4404 (2018).

[43] E. Aver, K. A. Olive, and E. D. Skillman, J. Cosmol. Astropart. Phys. 07 (2015) 011.

[44] A. D. Dolgov, S. H. Hansen, S. Pastor, S. T. Petcov, G. G. Raffelt, and D. V. Semikoz, Nucl. Phys. B632, 363 (2002).

[45] Y. Y. Y. Wong, Phys. Rev. D 66, 025015 (2002).

[46] K. N. Abazajian, J. F. Beacom, and N. F. Bell, Phys. Rev. D 66, 013008 (2002).

[47] S. Pastor, T. Pinto, and G. G. Raffelt, Phys. Rev. Lett. 102, 241302 (2009).

[48] G. Barenboim, W. H. Kinney, and W. I. Park, Phys. Rev. D 95, 043506 (2017).

[49] C. P. Ma and E. Bertschinger, Astrophys. J. 455, 7 (1995).

[50] A. Lewis and S. Bridle, Phys. Rev. D 66, 103511 (2002).
[51] R. Consiglio, P. F. de Salas, G. Mangano, G. Miele, S. Pastor, and O. Pisanti, Comput. Phys. Commun. 233, 237 (2018).

[52] N. Aghanim et al. (Planck Collaboration), Astron. Astrophys. 641, A8 (2020).

[53] F. Beutler, C. Blake, M. Colless, D. H. Jones, L. StaveleySmith, L. Campbell, Q. Parker, W. Saunders, and F. Watson, Mon. Not. R. Astron. Soc. 416, 3017 (2011).

[54] A. J. Ross, L. Samushia, C. Howlett, W. J. Percival, A. Burden, and M. Manera, Mon. Not. R. Astron. Soc. 449, 835 (2015).

[55] S. Alam et al. (BOSS Collaboration), Mon. Not. R. Astron. Soc. 470, 2617 (2017).

[56] R. J. Cooke, M. Pettini, and C. C. Steidel, Astrophys. J. 855, 102 (2018).

[57] R. J. Cooke, Astrophys. J. Lett. 812, L12 (2015).

[58] I. M. Oldengott and D. J. Schwarz, Europhys. Lett. 119, 29001 (2017).

[59] A. Bonilla, R. C. Nunes, and E. M. C. Abreu, Mon. Not. R. Astron. Soc. 485, 2486 (2019). 